\title{
Evaluation of Change in Air Gap Underneath the Garment for Various Pro-Longed Body Postures Using 3D Body Scanning
}

\author{
Emel MERT ${ }^{* 1,2}$, Agnes PSIKUTA ${ }^{1}$, Marie-Ange BUENO ${ }^{2}$, René M. ROSSI ${ }^{1}$ \\ ${ }^{1}$ Laboratory for Protection and Physiology, Empa - Swiss Federal Laboratories \\ for Materials Science and Technology, St. Gallen, Switzerland \\ ${ }^{2}$ Univ. Haute Alsace, Laboratoire de Physique et Mécanique Textiles, \\ Ecole Nationale Supèrieure d'Ingénieurs Haute Alsace, Mulhouse, France
}

http://dx.doi.org/10.15221/14.210

\begin{abstract}
Heat and mass transfer through the garment on wearer's body is affected by the change in the air gap thickness and the contact area more than by the material of the fabric. For thermally relevant evaluation of the thermal properties of clothing, e.g. during work or leisure time, it is important to analyse the air gap thickness and the contact area for prolonged, stationary and ergonomically correct body postures. A flexible manikin, which has similar body size and flexibility of joints as an adult male, was used to mimic realistic human body postures (standing and driving). To obtain accurate and reliable 3D scans, stands with fixations and the garment with easy openings were designed and Artec MHT 3D scanner was used. The presented study indicates that the distribution of air gap thickness and contact area between the body and the garment was depended on the posture of human body due to the gravitational force and the pressure from the chair on the flexible garment. The results of this study can contribute to an improvement of clothing design for functional garments and can be applied to heat and mass transfer modelling in clothing for various postures.
\end{abstract}

Keywords: air gap, contact area, 3D body scanning, prolonged postures, heat and mass transfer.

\section{Introduction}

People's expectation towards variety of clothing is to ensure a protection against harsh environmental conditions and thermal comfort [1]. This can be provided by maintaining balanced heat and mass transfer between the clothed body and its environment. The heat exchange processes in the clothing are affected by the change in the air gap thickness and the contact area more than by the material of the fabric. The thermal properties of the air layer are related to its size, which, in turn, depends on the form of the wearer's body (morphology, posture and the movement) [2], the mechanical properties of the fabric (fabric stiffness and shear resulting in fabric drapability), and garment design and size [3, 4]. Since the heat loss rate through the garment depends mostly on the shape and the thickness of air gap and the magnitude of the contact area between the human skin and the garment, it is important to accurately determine these parameters for variety of scenarios.

The stagnant air beneath and on the garment is an effective insulator against to heat and mass transfer in a prolonged body posture. Different methods have been used to evaluate the air gap volume using gas trace method [4-6], cylindrical mathematical method [8], three dimensional (3D) body scanning [9]. These methods contributed to overall evaluation of air gap and its thermal effect. However, there is only scarce information on local distribution of air layer and contact area and their effect on the heat and mass transfer through the garment. Therefore, further development has been done to evaluate local distribution of air gap thickness [3] and contact area between the body and the garment [10]. These studies offered an overview of the local distribution of air gap thickness and contact area over the body parts and are helpful for mathematical clothing modelling. However, the manikins used in these studies had an artificial standing straight position, which is ergonomically suited only for a short time. For thermally relevant evaluation of the thermal properties of clothing e.g. during work or leisure time, it is important to analyse the air gap thickness and the contact area for prolonged, stationary and ergonomically correct body postures.

The aim of this study is to accurately analyse the change in the distribution of air gap thickness and the contact area between human body and the garment due to various prolonged body postures using 3D scanning. A flexible manikin, which has similar body sizes and flexibility of joints of an adult male,

*Lerchenfeldstrasse 5, CH-9014 St. Gallen, Tel: +41 587657662 email: emel.mert@empa.ch 
was used to mimic realistic human body postures. To obtain accurate 3D scans and to avoid the disruption of the posture when undressing the manikin, stands with fixations and the garment with easy openings were designed. The selected manikin body postures corresponding to work configurations were chosen to be standing straight (as a reference) and driving. The results of this study can contribute to an improvement of clothing design for functional garments and can be applied to heat and mass transfer modelling in clothing for various postures.

\section{Material and Method}

\subsection{Manikin}

To mimic the prolonged postures of the human body, which are mostly confronted during work or leisure time, a flexible manikin with the approximate size of an adult male body has been selected (Figure 1, left). It was made of polyurethane with plastic coating (Polyform® $\mathrm{GmbH}$ \& Co. KG., Rinteln, Germany) with the following circumferences of $91 \mathrm{~cm}$ for chest, 77 for waist and 91 for hip. The manikin's posture can be changed by bending the joints, which are at the neck, shoulders, elbows, wrists, waist, hips, knees, ankles and fingers (Figure 1, middle). The bending angle of joints has limits, which are close to the needed angle for mimicking daily pro-longed postures of the human body.

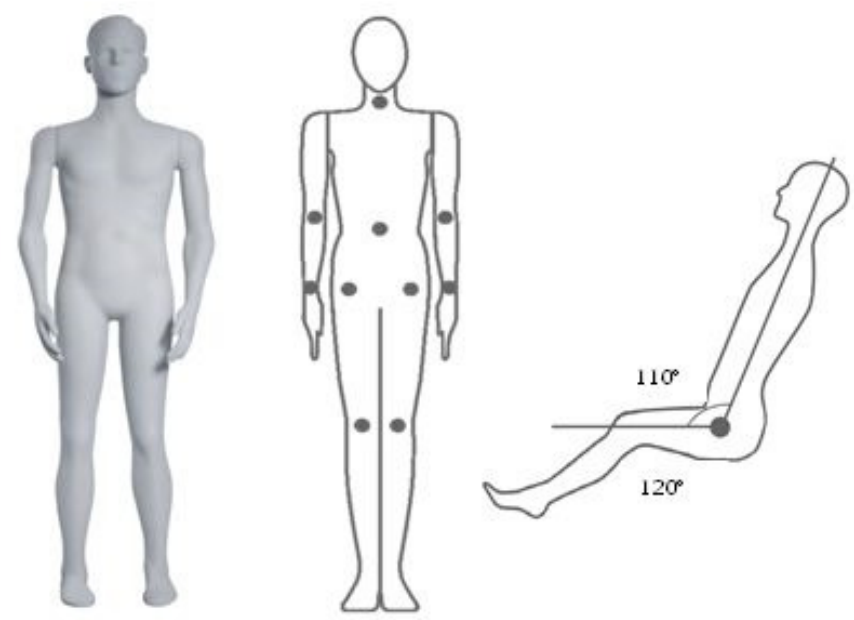

Fig. 1. Flexible manikin (left), the drawing of manikin with marking of bendable joints in standing posture (middle) and the drawing of manikin in driving posture (right)[Polyform®]

a)



b)

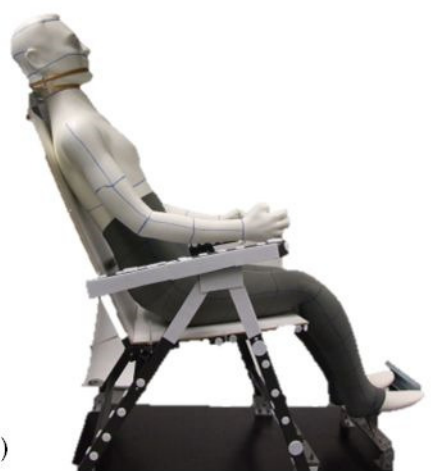

Fig. 2. Stands for a) standing with scanner's driver and b) driving posture 


\subsection{Postures}

For thermally relevant evaluation of the thermal properties of clothing, it is important to analyse the air gap thickness and the contact area for prolonged, stationary and ergonomically correct body postures. Therefore, in the present study, we proposed to evaluate the driving posture, which is often confronted during daily life as well as in many professions. To be able to make comparison with previous studies, standing posture was also selected as a reference. To avoid the disruption of the posture of the manikin in standing and driving postures, two different stands were designed with special locks. For standing posture a stand with locks at the foot and head were used (Figure 2a). To have better reliability of scans, a wooden reference was inserted in the proximity of the manikin. For driving posture, a stand with locks at foot and head were used (Figure $2 b$ ). The trunk-thigh angle and the thigh-calf angle were $110^{\circ}$ and $120^{\circ}$, respectively, which are average angles of drivers' lower limb [11] (Figure 1. right). To ensure this selected driving posture a chair with adjustable back were mounted on the stand (Figure 2b).

\subsection{Garment design}

In present study, plain knitted jersey, which contained $95 \%$ of cotton and $5 \%$ of spandex fibre, was used to sew the sweatpants. Fabric were washed with gentle washing programme at $40^{\circ} \mathrm{C}$ and dried to remove any foreign material, such as dust, soil, dye waste and the tensions of the manufacturing process from the fabric.

Casual sweatpants made of jersey knitted fabric were prepared for this study to represent the indoor clothing, which is used in daily life. The pattern of sweatpants was done in regular fit. Ease allowances of the garment calculated by subtracting the girth of manikin body from and the girth of garment at relevant body landmarks are given in Table 1.

Table 1: Ease allowances of garments

\begin{tabular}{|l|c|c|c|}
\hline Body part & $\begin{array}{c}\text { Measurements of Alf } \\
(\mathbf{c m})\end{array}$ & $\begin{array}{c}\text { Measurements of garment } \\
\text { (cm) }\end{array}$ & $\begin{array}{c}\text { Circumferential ease } \\
\text { allowance (cm) }\end{array}$ \\
\hline Hip & 91 & 108 & 17 \\
\hline Thigh & 51.5 & 52 & 0.5 \\
\hline Calf & 38.5 & 42 & 3.5 \\
\hline
\end{tabular}

Additionally, a zipper through the whole length of the leg was put in to dress and undress the manikin easily and avoid the disturbance on the manikin's posture (Figure 3).



Fig. 3. The front and side-view of regular fitted sweatpants and the zipper 


\subsection{Scanning procedure}

The Artec MHT (Artec Group, CA, USA), which is a hand-held 3D body scanner, was used to capture the clothed and nude manikin in standing and driving postures. Since the hand-held scanner has small view area $(536 \times 371 \mathrm{~mm})$, the scanner has to be moved around the manikin to be able to scan the large size of manikin. For scanning in the standing posture, a special driver was used, while for scanning in the driving posture, the scanner was used with guidance of a person.

It was difficult to obtain a complete scan of the manikin in driving posture owing to the limitations with the back of the chair. As a result, a large section of missing data at the posterior pelvis were occurred, where the manikin often in contact with the chair (Figure 4). Therefore, the posterior pelvis was excluded in the evaluation and considered as it has higher contact with the chair.

The 3D scans were post-processed by the dedicated software Geomagic Control 2014 (3D Systems $\AA$, SC, USA) to achieve the thickness of air gap and the magnitude of contact area. Scanning and post-processing procedure consist the following steps:

- Dressing the flexible manikin and mimicking the posture,

- Scanning dressed flexible manikin locally in various postures,

- Undressing the flexible manikin with the help of zippers,

- Scanning the nude flexible manikin locally in various postures,

- Cleaning and reorientation of the scans,

- Alignment of the scans,

- Geometrical determination of the distance between super-imposed surfaces recognized as air gap thickness and contact area.

\section{Results}

The division of the manikin's lower body into the body parts and the exemplary single coloured maps displaying the distribution of the air gap and the contact area in selected posture are shown in Figure 4. The average air gap thickness and the contact area and their standard deviations for different body parts in standing straight and driving postures are shown in Figure 5 and Figure 6, respectively.

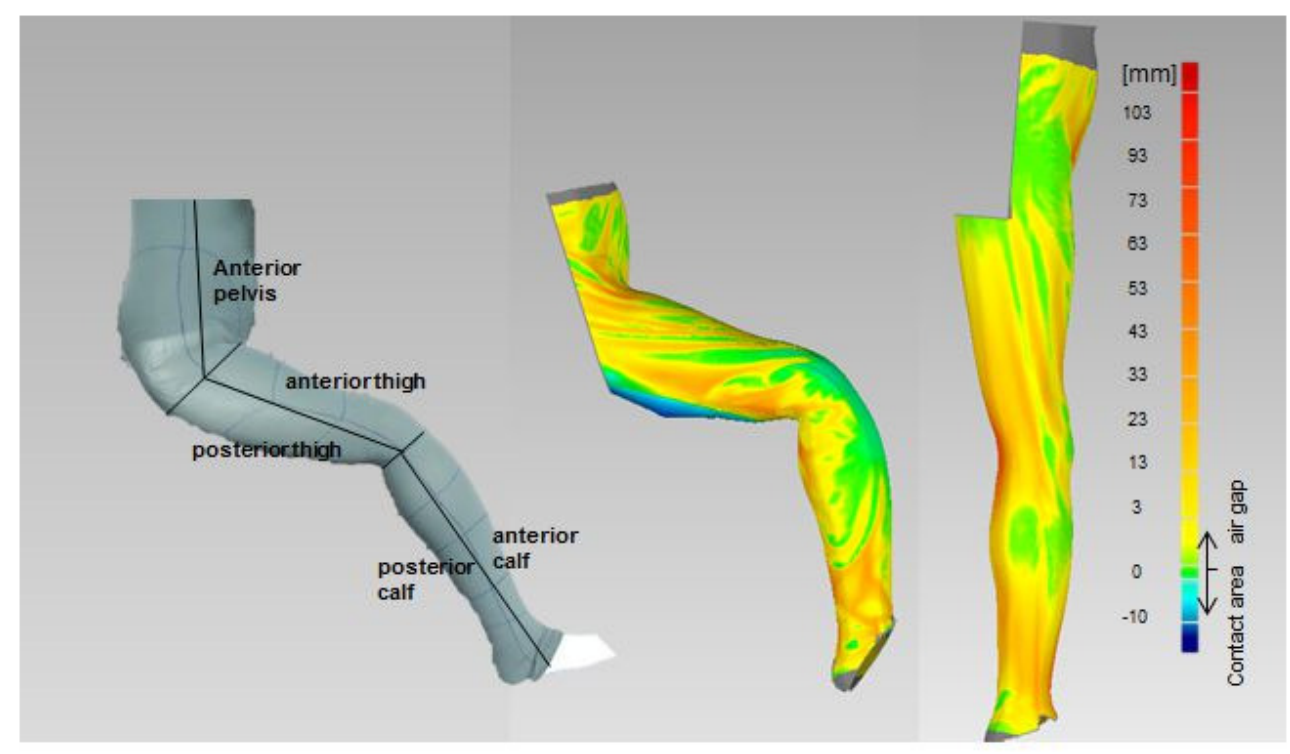

Fig. 4. Division of the manikin's lower body into the body parts (left) and exemplary single post-processed $3 D$ scans of driving (middle) and standing (right) postures indicating the contact area and the air gap thickness of the trousers 




Fig. 5. Average air gap thickness for different lower body parts in standing straight and driving postures

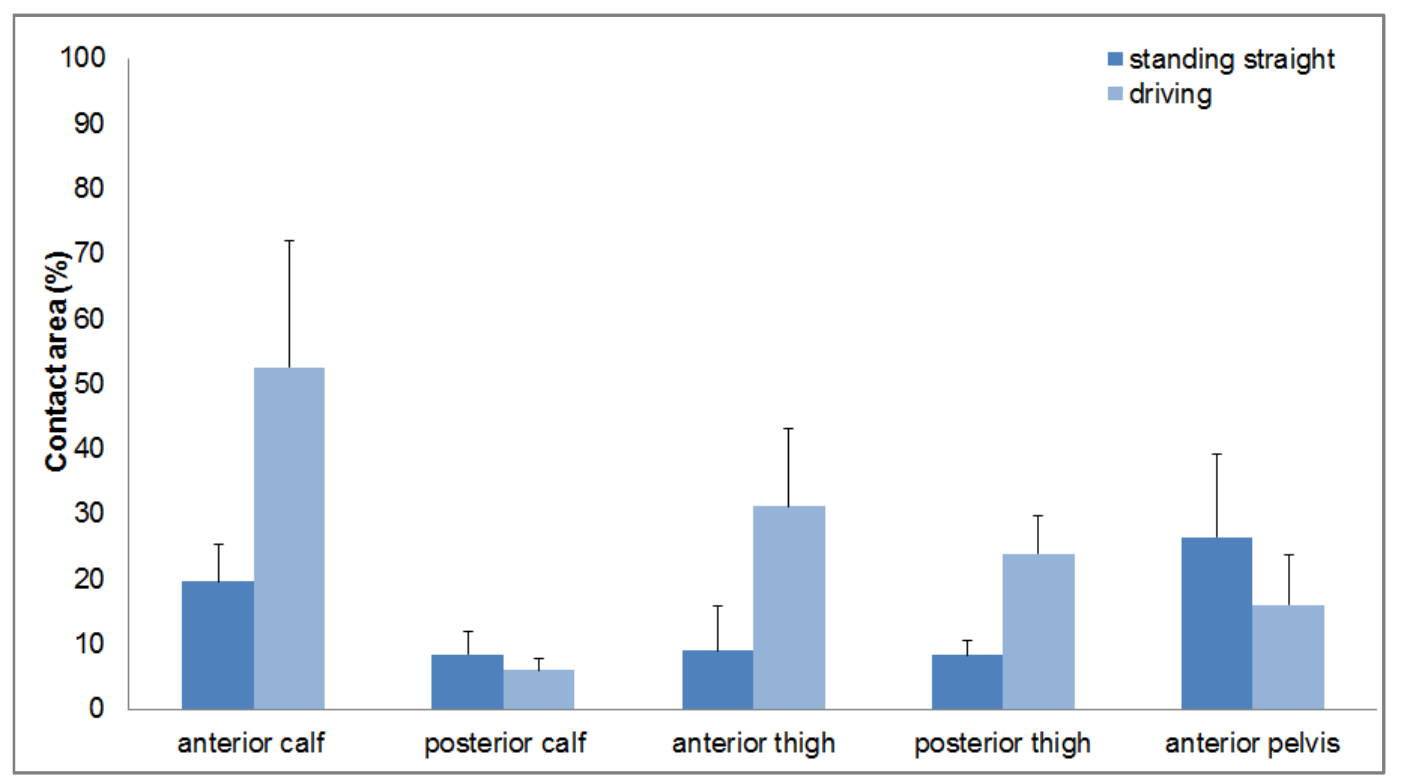

Fig. 6. Contact area for different lower body parts in standing straight and driving postures

\section{Discussion and conclusions}

Driving and standing postures, which are often confronted in daily and professional life, were compared to evaluate the change in the air gap thickness and the contact area for lower body. The presented study indicates that the distribution of air gap thickness and contact area between the body and the garment depended on the posture of human body and the effect of gravitational force. The change in air gap thickness and contact area for standing and driving postures varied between 0.6$3 \mathrm{~mm}$ and $2-33 \%$, respectively. The lowest and the highest change in air gap thickness were observed at posterior thigh $(0.6 \mathrm{~mm})$ and at anterior pelvis $(3 \mathrm{~mm})$, respectively. At the same time, the minimum and the maximum change in contact area occurred at posterior calf $(2 \%)$ and anterior calf $(33 \%)$, respectively (Figure 5 and 6 ). 

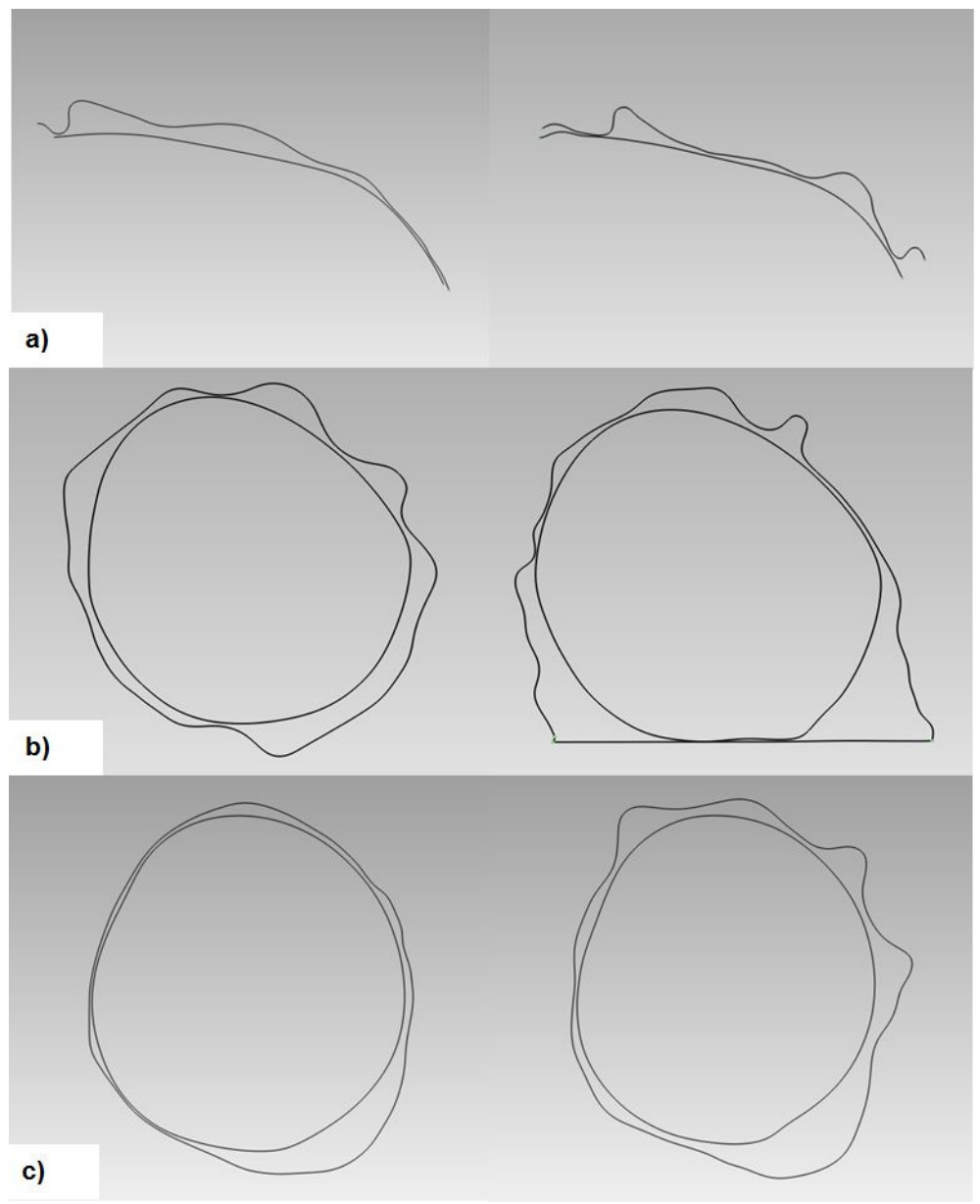

Fig. 7. Cross section of a) the anterior pelvis, b) the thigh and c) the calf in standing (left) and driving (right) postures

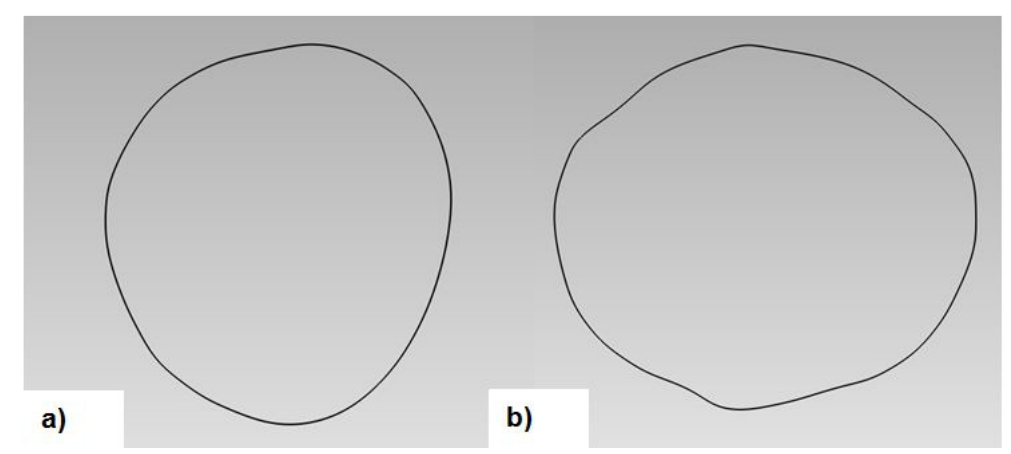

Fig. 8. Cross section of the knee of nude manikin in a) standing and b) driving postures

According to the Figure 5 and 6 , the change in air gap thickness and contact area for different body parts varied over body parts. The average air gap thickness for driving posture were lower than the average air gap thickness for standing postures at the entire lower body except for the posterior thigh. When the human sits on the chair, the pressure occurs between the leg and the chair. Additionally, the distortion on the garment owing to the change in posture can be seen in the cross sections of thigh in standing and driving postures, which are presented in Figure 7b. The garment fell over the posterior thigh due to the gravitationally force. Hence, the rest of the fabric was gathered at the both sides of the thigh. As a consequence of this effect, although the change in contact area was higher for posterior thigh, the observed change in air gap thickness was similar for both postures. For other body parts, such as anterior pelvis and calf, the distribution of air gap thickness and the contact area were as expected and evenly distributed around the body parts (Figure 7a and 7c). 
The thickness of air layer between $8-13 \mathrm{~mm}$ is considered as the threshold of free air circulation and increase the heat and mass transfer in clothing $[12,13]$. According to the results obtained from this study, in driving postures with regular fitted garment, the convection could be negligible since the air gap thickness were less than $13 \mathrm{~mm}$ for all lower body parts and regular pant design.

Since the shape and the orientation of the air layer between the garment and the body also plays an important role in the insulation of the garment, the effect of air layers at anterior thigh in driving and standing postures on the insulation of the garment is expected to be different. On anterior thigh in driving posture, the air layers were mostly in irregular shape owing to the two contrary applied forces on thigh from the bended hip and calf joints, whilst in standing posture the air layers were more homogenous (Figure 4, middle and right, and Figure 7b). Even though the thickness of air layer for both body parts was lower than $10 \mathrm{~mm}$, which is the boundary air layer thickness for taking place of the free air circulation underneath the garment, the heat loss through the anterior thigh in driving posture would be higher than the heat loss through the anterior thigh in standing posture due to the higher contact area in heterogeneous air layers.

The change in the thickness of air gap and the magnitude of contact area are also depended on the relocation of fat tissue due to increased pressure between the body parts and the pressure from the object (chair, desk) that touches to the skin. In presented study, it was not possible to evaluate the effect of the fat tissue on the change in air gap thickness and the contact area resulting from the different postures. In real case, the fat tissue would be deformed around region where the body would have pressure from other body part or an object. Since, it was not possible to scan nude and the clothed human without disturbing the posture, the evaluation of the distortion of the garment in various postures was done with a flexible manikin. When the limbs of the flexible manikin are bended, the joint area is deformed due to due to the pressure from neighbouring body parts on the polyurethane (Figure $8 \mathrm{a}$ and $8 \mathrm{~b}$ ). The manikin could mimic the posture of human realistically, however, for local small-scale deformations, e.g. muscles, fat tissue, are rather coarse approximations.

The interaction between the garment and the human body in different posture were evaluated using a flexible manikin and 3D scanning method. As a consequence, it was proven that the garment distortion occurred mostly on the body parts where the external forces, e.g. pressure from an object, pressure from neighbouring body part etc., were applied. This knowledge will contribute to the models of heat and mass transfer in clothing and the garment design for working postures. For further study, the detailed evaluation of the air gap thickness and the contact area in different garment design, body postures and morphology are required.

\section{Acknowledgments}

The authors wish to thank the members of the workshop, Jörg Gschwend, Pascal Luzi and Ernst Rüegg, at Empa for their prompt support.

\section{References}

[1] K. Parsons, "Human Thermal Environments: The effect of hot, moderate and cold environments on human health, comfort and performance", Second Edition, Taylor \& Francis, London, 2003.

[2] X. Li, Y. Wang, Y. Lu, "Effects of body postures on clothing air gap in protective clothing", in Journal of Fiber Bioengineering and Informatics, Vol.4, 2011, pp.277-283.

[3] T. Mah, G. Song, "Investigation of the contribution of garment design to thermal protection. Part 1: Characterizing air gaps using three-dimensional body scanning for women's protective clothing", in Textile Research Journal, Vol.80 No.13, 2010, pp.1317-1329.

[4] J. Frackiewicz-Kaczmarek, "Determination of the air gap thickness and the contact area under wearing conditions", Doctoral Thesis Universite de Mulhouse-Haute Alsace, 2013.

[5] G. W. Crockford, M.Crowder, S. P. Prestidge, "A trace gas technique for measuring clothing microclimate air exchange rates", in British Journal of Industrial Medicine, Vol.29, 1972, pp.378-386. 
[6] G. W. Crocford, H. A. Rosenblum, "The measurement of clothing microclimate volumes", in Clothing Research Journal, Vol.2, 1974, pp. 109-114.

[7] P. J. Sullivan, I. B. Mekjavic, N. Kakitsuba, "Determination of clothing microenvironment volume", in Ergonomics, Vol.30, 1987, pp.1043-1052.

[8] W. A. Lotens, and G. Havenith, "Calculation of Clothing Insulation and Vapor Resistance", in Ergonomics, Vol.34, 1991, pp.233-254.

[9] H. Daanen, K. Hatcher and G. Havenith, "Determination of clothing microclimate volume", Environmental Ergonomics - The Ergonomics of Human Comfort, Health, and Performance in the Thermal Environment, editors: Tochihara Y., Ohnaka T., 2005 pp.361-365.

[10] A. Psikuta, J. Frackiewicz-Kaczmarek, I. Frydrych R. Rossi, "Quantitative evaluation of air gap thickness and contact area between body and garment", in Textile Research Journal, vol.82, 2012, pp.1405-1413, doi:Doi 10.1177/0040517512436823

[11] X. Wang and M. I. Bullock, "Pedal Operation, Preferred Postures for Driving", in Working Postures and Movements-Tools for Evaluation and Engineering, Edited by N. J. Delleman, C. M. Heslegrave and D. B. Chaffin, CRC Press, 2004.

[12] J.L. Spencer-Smith, "The physical basis of clothing comfort part 2: heat transfer through dry clothing assemblies", in Clothing Research Journal, Vol.5, 1977, pp.3-17.

[13] B. Cain and B. Farnworth, "Two new techniques for determining the thermal radiative properties of thin fabrics", in Journal of Building Physics, Vol.9, 1986, pp.301-322. 